to the percentage of satisfactory smears or the rate of detection of abnormal cells.

Conclusion: Our results support the efficacy of spatulacytobrush for cervical cancer screening.

Poster (C06)

Cervical Cancer

https://doi.org/10.3802/jgo.2021.32.S1.c06

\section{Utility of radiomics for predicting patient local control in cervical cancer with chemoradiotherapy}

\section{Wei Jiang, ${ }^{1}$ Haowen Pang, ${ }^{2, *}$ Han Chen ${ }^{2}$}

'Department of Radiotherapy, Yantai Yuhuangding Hospital, Yantai, China ${ }^{2}$ Department of Oncology, The Affiliated Hospital of Southwest Medical University, Luzhou, China (8680003@qq.com)

Objective: The aim of this study was to develop a predictive model combining radiomic features with clinical characteristics for predicting local control (LC) in cervical cancer patients receiving intensity modulated radiotherapy (IMRT).

Methods: This was a retrospective analysis of 161 patients with cervical cancer treated with IMRT, using data acquired between May 2012 and March 2017. We extracted the radiomic features from computed tomography (CT) scans. Least absolute shrinkage and selection operator regression was used to filter the extracted radiomic features and reduce the dimensionality of the data. Rad-score was calculated by the selected radiomic features. Multivariate Cox-regression hazard models was established to analyze the LC of cervical cancer patients, and a Nomogram prediction model based on Rad-score and clinical characteristics was used to demonstrate the prediction model. The prediction reliability was evaluated by the area under the receiver operating characteristic curve and Harrell's concordance index. Decision curve analysis evaluates the application value of the Nomogram. The heat map shows the relationship between radiomics features and clinical characteristics. According to the cut-off value of Radscore, all patients were classified as low-risk or high-risk.

Results: A total of 5 radiomic features and 2 clinical characteristics were extracted for analysis. A combination of the Rad-score and clinical characteristics resulted in better performance for the estimation of 5-year LC (area under the curve $[A U C]=0.780$; confidence interval $[\mathrm{CI}]=0.690-0.870)$ than that with clinical characteristics alone (AUC $=0.680 ; \mathrm{CI}=0.590-0.770$ ). These patients were divided into high-risk and low-risk groups according to the cut-off value of Rad-score.

Conclusion: This study shows that the combination of CT extracted radiomic features and clinical characteristics has a good potential for evaluating LC in patients with cervical cancer treated with IMRT.
Poster (C07)

Cervical Cancer

https://doi.org/10.3802/jgo.2021.32.S1.C07

\section{Pretreatment lymphocyte counts as independent prognostic factors in patients with locally advanced cervical cancer treated with concurrent chemoradiotherapy}

Ekasak Thiangphak, Korrawit Pruegsanusak, Rakchai Buhachat ${ }^{*}$

Songklanagarind Hospital, Hat Yai, Thailand (brakchai@medicine.psu.ac.th)

Objective: To evaluate the association between pretreatment total lymphocyte counts (TLC) and survival in locally advanced cervical cancer patients treated with concurrent chemoradiation (CCRT). Methods: A Retrospective analysis of 789 patients diagnosed cervical cancer stage IIB to IVA who treated with primary CCRT from January 2011 to December 2015 was performed. Pretreatment TLC was evaluated for an association with 5-year disease free survival (DFS) and overall survival (OS) rates. Results: Seven hundred and eighty-nine patients were included in the study. There were 52 patients had pretreatment TLC $<1,000$ cells $/ \mathrm{mm} 3$. The median pre-treatment TLC in groups TLC $<1,000$ cells $/ \mathrm{mm} 3$ and $>1,000$ cells/mm3 were 573.9 cells/ mm3 (range $=350.9-827.7$ cells $/ \mathrm{mm} 3$ ) and 2,211.3 cells/mm3 (range $=1,751.3-2,785.8$ cells/mm3) respectively. Patients in pretreatment TLC $<1,000$ cells/mm3 group had fewer number of treatment response and trend to present in more advance stages of disease and larger tumor size. The 5-year DFS and OS rates were significantly higher in patients with pretreatment TLC $>1,000$ cells/ $\mathrm{mm} 3$ than the counterparts $(67.7 \%$ vs. $35.4 \%$ [ $\mathrm{p}<0.0001], 57.6 \%$ vs. $25.7 \%[\mathrm{p}<0.0001])$. Multivariate analysis showed pretreatment TLC $>1,000$ cells/mm3 was independent predictors of DFS (hazard ratio $[\mathrm{HR}]=0.39 ; 95 \%$ confidence interval $[\mathrm{CI}]=0.26-0.59 ; \mathrm{p}<0.001)$ and OS (HR=0.59; 95\% CI=0.42-0.84; p=0.006) after adjusted with age, stages of disease and tumor size.

Conclusion: Pretreatment TLC were associated with treatment response, and also the survival outcome in patients with locally advanced stage cervical cancer treated with definite CCRT.

Poster (C08)

Rare Tumors \& Metastatic Tumors

https://doi.org/10.3802/jgo.2021.32.S1.c08

\section{Primary diffuse large B-cell lymphoma of uterine cervix presenting with bulky cervical mass: a case report ana review}

\footnotetext{
A-jaree Senthong, 'Suwapee Watcharahirun

Surin Hospital, Surin, Thailand (ajaree0348@gmail.com)
} 
Objective: Primary lymphoma of female genital tract is rare and only $0.3 \%-1.5 \%$ of extra-nodal non Hodgkin' lymphomas. The cervix is the second common site in literatures review. The diagnosis is difficult due to submucosal stromal lesion and nonspecific manifestation without constitutional 'B-cell' symptoms. Combined chemotherapy (CHOP) is mainstay for treatment. Methods: Review of a case and literatures.

Results: A 39 years-old woman presented to the gynecological clinic with repeated vaginal discharge, pelvic discomfort for 4 months. Her annual Pap test was low-grade squamous intraepithelial lesion. During pelvic examination, a large $6 \times 6$ $\mathrm{cm}$. Infiltrative bulky cervical mass was visualized. Rectovaginal examination found cervical tumor extended to parametrial tissue both sides, fixating to the left pelvic sidewall. The colposcopic biopsy result was cervicitis. The deep excisional procedure was performed and result was atypical lymphoid proliferation. Immunohistochemistry (IHC) were positive for CD20, BCL6 (90\%), Ki 67 (50\%-60\%). IHC were negative forAE1/AE3. The diagnosis was diffuse large B-cell lymphoma (WHO Classification 2008). The magnetic resonance imaging show large cervical mass $4.7 \times 4.9 \times 5.3 \mathrm{~cm}$ at posterior cervix with invasion to stroma, left parametrium and protrusion into upper vagina. No upper abdominal metastasis and lymphadenopathy were observed. Bone marrow biopsy result was hypocellular and compatible with Ann Arbor stage I. The patient was sent to hematologist and received R-CHOP Cyclophosphamide, Doxorubicin, Vincristine, Prednisone, and Rituximab) for 8 cycles and achieved CR. The patient is remaining remission after 55 months follow up.

Conclusion: Primary lymphoma of cervix is extremely rare and deep excisional procedure was useful for diagnosis. CHOP is primary treatment and improved survival outcome.

Poster (C09)

Gynecologic Cancer Screening

https://doi.org/10.3802/jgo.2021.32.S1.c09

\section{Temporary elevation of serum squamous cell carcinoma antigen during follow-up and its clinical significance}

\author{
Min Feng, Ting-Chang Chang, Hung-Hsueh Chou, \\ Chyong-Huey Lai \\ Linkou Chang Gung Memorial Hospital, Taoyuan City, Taiwan
} (karen23154@hotmail.com)

Objective: We evaluated the role of serum squamous cell carcinoma antigen (SCC-Ag) in predicting recurrence after primary treatment.

Methods: Of 4,814 cervical cancer patients at Chang Gung Memorial Hospital Linkou Medical Center in 2000-2018, 2,206 patients had SCC-Ag testing at diagnosis.

Results: Excluding 27 patients with incomplete medical documents, 1,091 (50.1\%) showed elevated SCC-Ag, in whom 1,016 was squamous cell carcinoma. $81.8 \%$ cases with advanced stage (FIGO IIB or more), and $61.6 \%$ with a large tumor in early stage (IB3, IIA2) had elevated SCC-Ag. Seven hundred sixty-nine (75.7\%) of the 1,016 patients had CR to primary treatment, and their SCC-Ag returned to normal. A total of 207 patients had re-elevation of SCC-Ag at follow-up, in which 110 cases had recurrence. SCC-Ag re-elevation before imaging diagnosis was noted in $79(71.8 \%)$ of the recurred, $14(12.7 \%)$ patients had concurrent SCC-Ag re-elevation and imaging diagnosis of recurrence, another 11 (10.0\%) had recurrence before SCC re-elevation. Four had recurrences with normal SCC-Ag. 97 of the 207 patients with re-elevated SCC showed NED throughout follow-up, and their SCC-Ag returned to normal within six months. Forty-four (45.3\%) of the SCC elevation may be attributed to impaired renal function. Four patients had skin lesions. The cause of elevation in another 47 (48.5\%) could not be recognized. The overall sensitivity of SCC-Ag for detecting recurrence is $86.8 \%$, specificity $85.2 \%$, PPV $50.8 \%$, and NPV $97.4 \%$.

Conclusion: Serum SCC-Ag levels can be a useful tool for screening for cancer recurrence after primary treatment in patients with cervical squamous cell carcinoma.

Poster (C10)

Cervical Cancer

https://doi.org/10.3802/jgo.2021.32.S1.C10

\section{Transcription factor homeobox D9 drives the malignant phenotype of HPV18-positive cervical cancer cells via binding to the viral early promoter}

\section{Guanliang Chen, Takashi Iwata," Ryotaro Imagawa, Hiroshi Nishio, Yuki Katoh, Masaki Sugawara, Tomoya Matsui, Daisuke Aoki}

Keio University School of Medicine, Tokyo, Japan (iwatatakashi@1995.jukuin.keio.ac.jp)

Objective: Transcription factor homeobox D9 (HOXD9) is highly expressed in cervical cancer. Previously we reported HOXD9 bound to the P97 promoter of HPV16, and regulated early oncogenes E6 and E7. In this study, we investigated whether HOXD9 regulated the P105 promoter of HPV18 and examined the role of HOXD9 in intracellular signaling of cervical cancer.

Methods: HOXD9 knockdown HPV18 positive cervical cancer cell lines SKG-1 and Hela were used in this study. Cell cycle 